\title{
Prevalence of overweight and obesity among children and adolescents in Saudi Arabia
}

\author{
M. Almughamisi, T. George and S. Harding \\ Nutritional Sciences Research Division, King's College London, London SE1 9NH, UK
}

Childhood obesity is known as one of the most serious public health problems of the $21^{\text {st }}$ century. The prevalence of obesity remains high in several high-income countries although the level may have reached a plateau. ${ }^{(2)}$ The problem is global and from the scarce data available is an increasing problem for the low-income and middle-income countries ${ }^{(1)}$. In preparation of developing a school-based obesity prevention intervention programme for adolescent girls in Jeddah, we reviewed the literature on the prevalence of overweight and obesity among children and adolescents in Saudi Arabia, and the association with dietary habits, physical activity and socioeconomic circumstances.

A comprehensive search was conducted using different electronic databases, Embase, Global Health, Ovid Medline, Web of Science, PubMed, and Science Direct. An additional search was run through some website to avoid missing papers on database included (Google scholar and Saudi Journal of obesity). The search was performed using a variety of keywords such as Child/childhood OR Adolescents/Teens AND overweight OR Obesity AND Saudi Arabia.

Two hundred and fifteen papers were identified from the search and 180 were excluded. Of the 35 studies that did meet the inclusion criteria, 11 were classified as good quality, 7 as medium quality and 17 as poor quality. Studies classified as medium quality were mainly due to non-reporting of power calculations and of response rates. Studies classified as poor were mainly due to small sample sizes, poor description of study design and use of non-validated/referenced questionnaires.

The findings suggest an increase in childhood/adolescent obesity in the 2000s (Figure), that about one third of children/adolescents were overweight or obese by adolescence in the 2005 and 2011 national surveys, and that the steep rise in obesity and overweight occurred between ages 5 and 10 years.

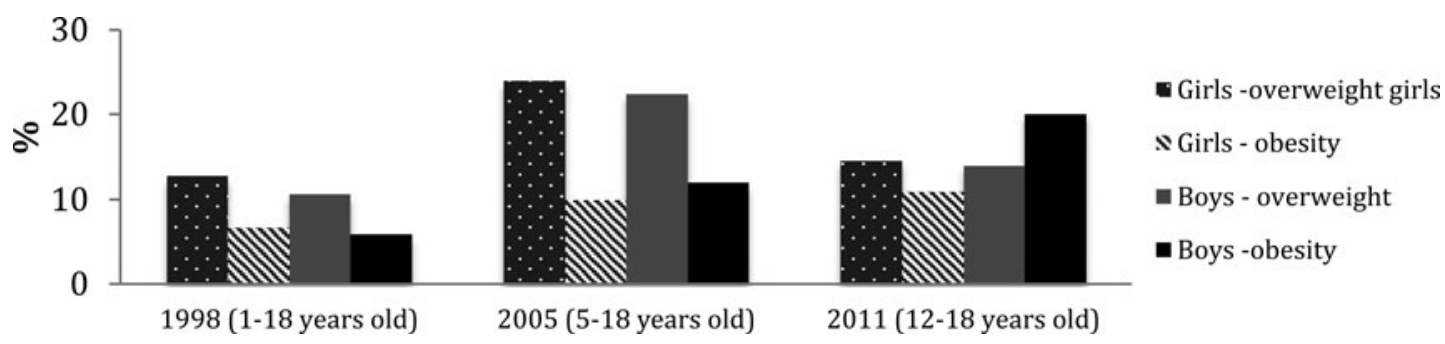

Fig. 1. Prevalence of overweight and obesity in Saudi Arabia (National Surveys).

Sugar sweetened beverages, frequency of eating away from home and skipping breakfast were associated with overweight and obesity. Low levels of physical activity were associated with overweight and obesity. The prevalence of overweight and obesity were more common among children with mothers who worked than among those who did not. Low levels of physical activity and poor food choice were a common feature in many studies regardless of gender, which provides opportunities for school based interventions.

1. Sahoo K, Sahoo B, Choudhury AK, Sofi NY, Kumra R \& Bhadorai AS (2015) Childhood obesity: causes and consequences. Journal of family medicine and primary care $\mathbf{4}, 187$.

2. Ogden CL, Carroll MD, Kiy BK \& Flegal KM (2014) Prevalence of childhood and adult obesity in the United States, 2011-2012. JAMA 311, 806-14. 